\title{
Desempenho de agências bancárias no Brasil: aplicando análise envoltória de dados (DEA) $a$ indicadores relacionados às perspectivas do BSC
}

\section{Performance of bank branches in Brazil: applying data envelopment analysis (DEA) to indicators related to the $\mathrm{BSC}$ perspectives}

\author{
Marcelo Álvaro da Silva Macedo \\ Ana Carolina Thomaz de Almeida Monteiro Barbosa \\ Glaydson Teixeira Cavalcante
}

\begin{abstract}
Resumo
Este estudo consiste na apresentação e discussão de uma metodologia com base na análise envoltória de dados (DEA), que procura promover uma visão do desempenho de unidades de negócio, neste caso 50 agências de um grande banco brasileiro, com base em dados do segundo semestre de 2006, tendo como variáveis críticas de sucesso seis indicadores que representam as perspectivas do BSC utilizadas por essas agências bancárias. Os resultados mostraram que apenas dez agências são tidas realmente como eficientes, servindo assim de padrão de referência (benchmark) para as outras. Além disso, o indicador da perspectiva financeira se apresenta com um grande potencial de melhoria para incrementar o desempenho multicriterial das agências sob análise e, por conseguinte, do banco como um todo.
\end{abstract}

Palavras-chave: Desempenho organizacional; BSC; Unidades de negócio; Agências bancárias; DEA.

\begin{abstract}
This paper introduces and discusses a methodology based on Data Envelopment Analysis (DEA), which attempts to provide an overall view of business units' performances. In this case, about fifty branches of a big Brazilian bank were focused on, with basis on data from the second semester of 2006, having as critical variables of success six indicators that represent BSC perspectives used by those bank branches. Results showed that only ten branches were really efficient, working as benchmarks to the others. Moreover, the financial perspective measure showed great potential to improve the multicriteria performance of the branches under analysis and, therefore, of the bank as a whole.
\end{abstract}

Key words: Organizational performance; BSC; Business units; Bank branches; DEA.

A avaliação de desempenho é algo importante na gestão de empresas de qualquer setor de atuação. Isso porque, dessa forma, a administração se torna capaz de monitorar, comparar e até mesmo corrigir o desempenho da organização. Assim, a avaliação do desempenho é fundamental para a sobrevivência da organização num ambiente competitivo.

A análise de desempenho de uma organização é sempre algo passível de muitas discussões. Questionamentos de quais indicadores utilizar e como consolidá-los de forma a 
estabelecer um critério justo de avaliação de performance estão presentes em vários estudos e no dia a dia das empresas.

Porém, não existe um modelo de avaliação de desempenho organizacional que seja único para todas as variáveis do mundo empresarial. No entanto, alguns métodos são capazes de considerar aspectos diversificados, assumindo, assim, um papel crucial na avaliação da performance empresarial, como a medida de eficiência utilizada neste trabalho.

Nesse contexto de análise multicriterial do desempenho organizacional é necessário destacar o balanced scorecard. O BSC foi desenvolvido num ambiente em que melhorias de desempenho exigiam mudanças também nos sistemas de mediação e gestão utilizados pelas empresas, pois era impossível atingir a excelência empresarial controlando apenas as medidas financeiras do desempenho passado, visto que os executivos necessitavam de indicadores sobre vários aspectos do ambiente e desempenho organizacional. Assim, o BSC pôde se apresentar como uma alternativa que representava um sistema de mensuração com indicadores financeiros e não financeiros, cujas medidas focalizavam o desempenho organizacional sob quatro perspectivas: financeira, do cliente, dos processos internos e de aprendizado e crescimento, equilibrando-se entre medidas objetivas, de resultado e facilmente quantificáveis, e vetores subjetivos, até certo ponto arbitrários, das medidas de resultado.

Porém, um questionamento sempre presente, quando se discute a questão de métodos ou metodologias multicriteriais de análise e avaliação do desempenho organizacional, é como consolidar múltiplas perspectivas de modo a obter uma visão do desempenho conjugado oriundo delas. Nesse sentido, pode-se utilizar uma metodologia multicriterial que consegue consolidar diversos vetores de desempenho em um único, denominada análise envoltória de dados (DEA).

A medida de desempenho DEA é de tal ordem que consolida em um único indicador as diversas perspectivas consideradas para análise da performance empresarial, através de uma modelagem com base em programação matemática que relaciona indicadores de tipo "quanto maior, melhor" e outros do tipo "quanto menor, melhor".

É nesse contexto de análise e avaliação do desempenho organizacional que se apresenta este trabalho, que tem como objetivo avaliar o desempenho de 50 agências bancárias de um grande banco brasileiro, com base em dados do segundo semestre de 2006, aplicando DEA a seis indicadores que representam as múltiplas perspectivas do BSC. 


\section{Desempenho organizacional e DEA}

A globalização e a abertura dos mercados fizeram com que as organizações sofressem mudanças para se adaptarem ao novo quadro mundial. Nesse quadro, em que a competitividade é um fator determinante, os sistemas de medição de desempenho se tornaram indispensáveis.

A mensuração de desempenho é definida por Neely (1998) como o processo de quantificar a eficiência e a efetividade de ações passadas, através da aquisição, coleta, classificação, análise, interpretação e disseminação dos dados apropriados. Ao final dessa trajetória, os sistemas de medição fornecem o grau de evolução, estagnação ou involução dos processos e atividades das empresas sob análise, gerando informações importantes e a tempo de que as ações preventivas e/ou corretivas sejam tomadas. Como o progresso da empresa é monitorado através desses sistemas, seus eventuais erros são corrigidos, aumentando as possibilidades de que a empresa conquiste suas metas e objetivos.

De acordo com Macedo et al. (2006), os processos de mensuração de desempenho se desenvolvem a partir da seleção de indicadores críticos de desempenho específicos para cada empresa. Entretanto, a maior dificuldade dos gestores está na escolha desses indicadores. O principal meio de definir esses indicadores seria, primeiramente, definir onde a organização se encontra e aonde quer chegar, para posteriormente definir os mecanismos que farão com que isso seja possível. Tais processos são permanentes e repetitivos, sendo a frequência de suas medições dependente da atividade a ser medida.

Para Slack et al. (2002), toda organização precisa, de alguma forma, de medidas de desempenho como um pré-requisito para melhoramento. No entanto, as práticas de medição de desempenho variam entre as empresas. Depois da mensuração de desempenho, usando, normalmente, um conjunto de medidas parciais, a organização precisa fazer um julgamento sobre sua performance. Esse julgamento, segundo os autores, envolve a comparação do nível de desempenho atingido com algum tipo de padrão.

Quando as empresas utilizam o mercado como parâmetro de comparação, segundo padrões de desempenho da concorrência, é necessário usar uma abordagem denominada benchmarking. Essa abordagem consiste na performance relativa das empresas em função de um conjunto de competidores previamente selecionados (SLACK et al., 2002).

Os sistemas de mensuração de desempenho são essenciais para que as organizações possam competir no mercado global. Porém, as avaliações feitas em caráter relativo, ou seja, mensurando a eficiência da organização em relação, por exemplo, a seu ambiente competitivo, são geradoras de resultados potencialmente consistentes. Isso ocorre porque informam o posicionamento da empresa perante a referência e as demais empresas. 
Não existe possibilidade de reduzir a complexidade do desempenho de um negócio a um único indicador, havendo necessidade real de aplicar diversos indicadores para atingir os mais variados aspectos nos quais a estratégia de negócio se realiza (SLACK et al., 2002) Assim, um programa de avaliação de desempenho eficiente deve incluir todos esses elementos considerados críticos de sucesso. Isso quer dizer que todos os elementos relevantes das empresas, de maneira individual, precisam ser, de alguma forma, mensurados para permitir análises comparativas.

São inúmeras as variáveis do mundo empresarial passíveis de mensuração. No entanto, não existem, ainda, modelos de avaliação de performance únicos para todas essas variáveis.A solução encontrada pelos gestores é a utilização de uma série de metodologias de avaliação capazes de tratar os diferentes elementos da organização. Essas técnicas produzem resultados isolados, uma vez que não consideram a multiplicidade de fatores numa única análise.

Nesse sentido, a análise envoltória de dados (DEA) apresenta-se como uma medida de desempenho capaz de comparar a eficiência de várias unidades operacionais similares mediante a consideração explícita do uso de suas múltiplas entradas para a produção de múltiplas saídas. Dessa forma, essa metodologia faz com que a decisão fique orientada por um único indicador construído a partir de várias abordagens de desempenho diferentes. Portanto, como congrega diversas perspectivas, a técnica promove uma melhor percepção da performance organizacional.

Segundo Lins e Meza (2000), a metodologia DEA, que teve origem no trabalho de Farrel (1957), caracteriza-se como uma técnica não paramétrica que permite lidar com várias saídas (outputs) e entradas (inputs), para analisar, comparativamente, unidades independentes no que se refere ao seu desempenho, ou seja, à eficiência de cada unidade.

São várias as formulações dos modelos de DEA encontradas na literatura, conforme Charnes et al. (1994), mas dois modelos básicos DEA são geralmente usados nas aplicações. O primeiro modelo, chamado de CCR (CHARNES; COOPER; RHODES, 1978), também conhecido como CRS (Constant returns to scale), avalia a eficiência total, identifica as DMUs eficientes e ineficientes e determina a que distância da fronteira de eficiência estão as unidades ineficientes. O segundo, chamado de modelo BCC (BANKER; CHARNES; COOPER, 1984), também conhecido como VRS (Variable returns to scale), utiliza uma formulação que permite a projeção de cada DMU ineficiente sobre a superfície de fronteira (envoltória) determinada pelas DMUs eficientes de tamanho compatível.

No caso das formulações, além das da escolha entre CRS e VRS existe a necessidade de fixação da ótica de análise (orientação input ou orientação output). Macedo et al. (2006) dizem que a abordagem DEA baseada nas entradas (inputs) busca maximizar as quantidades de produtos, isto é, maximizar uma combinação linear das quantidades dos vários produtos da 
empresa. Já para uma abordagem baseada nas saídas (outputs), busca-se minimizar as quantidades de insumos, isto é, minimizar uma combinação linear das quantidades dos vários insumos da empresa.

\section{Balanced scorecard (BSC)}

O processo de globalização que se consolidou nos últimos anos trouxe uma série de novos desafios para as empresas. Um desses desafios, e talvez o mais importante deles, foi a busca por mecanismos de mensuração de desempenho que não se limitassem a aspectos financeiros, mas englobassem uma amplitude de parâmetros que, de fato, caracterizassem a performance das instituições, assim permitindo que elas fossem mais competitivas.

Pace et al. (2003) confirmam essa tendência de utilização de medidas não financeiras na busca do melhor entendimento dos modelos de gestão. Os autores ressaltam, ainda, a ligação dessas medidas não-tradicionais com os recentes desenvolvimentos na área de planejamento estratégico.

O BSC - balanced scorecard - foi criado com a finalidade de desenvolver um novo grupo de medidas de desempenho, a partir da percepção de que a gestão empresarial baseada somente nas medidas financeiras estava se tornando obsoleta e que a dependência dessas medidas estava prejudicando a capacidade da empresa de criar valor econômico futuro (KAPLAN; NORTON, 1997).

Segundo Atkinson et al. (2000), o BSC reflete a primeira tentativa sistemática de desenvolver um sistema de avaliação de desempenho que enfoque os objetivos organizacionais, a coordenação do processo decisório e a provisão de uma base para o aprendizado organizacional. Esse sistema avalia os aspectos do desempenho organizacional que sinalizam o que os gestores acreditam serem os direcionadores da performance empresarial, ou seja, as causas da eficiência ou ineficiência da empresa.

Assim, a metodologia se apresenta como uma proposta de mensuração estruturada que complementa a abordagem tradicional de avaliação de resultado, integrando a perspectiva financeira às perspectivas representativas dos processos internos, da relação entre empresa e cliente e da capacidade da organização no que se refere ao aprendizado e crescimento. Essas perspectivas, segundo os autores acima citados, podem ser assim descritas:

- Perspectiva financeira: as medidas financeiras, na abordagem BSC, deixam de ser o foco principal, mas são preservadas devido à sua importância;

- Perspectiva dos processos internos: está relacionada à identificação dos processos críticos nos quais a empresa deve alcançar a excelência, oferecendo propostas de valor 
que atraiam e retenham clientes em segmentos-alvo de mercado e satisfaçam, certamente, às expectativas financeiras dos acionistas;

- Perspectiva do cliente: está relacionada à identificação de segmentos de clientes e mercados nos quais a organização competirá e as medidas de desempenho nesses segmentos-alvo;

- Perspectiva do aprendizado e crescimento: está relacionada à identificação da infraestrutura que a empresa deve construir para gerar crescimento e melhoria no longo prazo.

Através do balanced scorecard, Kaplan e Norton (1997) afirmam que é possível avaliar até que ponto as unidades de negócio de uma empresa geram valor para os clientes atuais e futuros, e como podem aperfeiçoar suas capacidades internas, sistemas e procedimentos, e fazer investimentos necessários em pessoal, visando a melhorias no desempenho.

Davis e Albright (2004) investigaram a capacidade de o BSC incrementar a performance financeira em uma organização. Os pesquisadores verificaram que a unidade onde o BSC foi implementado possuía desempenho financeiro superior à unidade em que a ferramenta não havia sido implementada.

Kimura e Suen (2003) enfatizam a importância do BSC para as empresas, justamente porque por meio dele é possível identificar o desempenho empresarial através da consideração de resultados de rentabilidade e custos, assim como de indicadores de atendimento das necessidades dos clientes, de eficiência de processos internos e de potencial de aprendizado e crescimento da organização.

Além disso, como vantagem da metodologia, pode-se citar a possibilidade de inclusão de outros indicadores específicos e até mesmo de perspectivas adicionais de grande importância para o segmento de uma determinada empresa, o que proporciona uma análise mais completa e exata.

Para Goldszmidt (2003), o balanced scorecard apresenta atualmente elevado nível de aplicação e importantes resultados têm sido atingidos pelas organizações que o utilizam. Entretanto, há relatos de tentativas fracassadas de implementação do BSC, o que leva a crer que poderiam existir fatores críticos para que ele seja bem-sucedido.

Assim como o autor supracitado, muitos outros ressaltam, além dos benefícios, as limitações dessa metodologia. Conforme Norreklit (2000), apesar de permitirem a inclusão de novas perspectivas na abordagem BSC, Kaplan e Norton (1997) não discutem como essas perspectivas adicionais deveriam ser incorporadas à metodologia, nem explicam como ficariam as relações de causa e efeito entre as medidas de desempenho. 
Epstein e Manzoni (1998) questionam a habilidade de os executivos articularem uma visão clara e comum da estratégia da empresa, capaz de permitir a construção do BSC. Esses autores consideram que a definição da estratégia pode ser um processo difícil para os executivos de uma grande empresa. Em alguns casos, a estratégia não está bem definida e, em outros, os membros da alta administração possuem opiniões diferentes sobre qual deve ser a estratégia da organização.

Além das barreiras citadas acima, algumas falhas estruturais e organizacionais dificultam a implantação do BSC. Dentre as falhas estruturais, conforme Galas (2004), têm-se medidas que mostram a eficiência passada das estratégias da empresa que são úteis para controlar, mas não para comunicar o seu andamento, além de não permitir ajustes. Em relação às falhas organizacionais, o autor cita: delegação do BSC a uma equipe de nível gerencial médio; utilização de indicadores inadequados à empresa; e criação de sistemas de informação para geração de indicadores.

Schneiderman (1999) reforça a ideia de que o BSC impõe uma mudança organizacional, afirmando que as organizações somente mudam quando empregados compartilham a propriedade das metas e dos meios. Para esse autor, as razões para a maioria das falhas na implementação do BSC são:

- As variáveis não financeiras são identificadas incorretamente como direcionadores primários da satisfação futura dos stakeholders.

- As medidas são mal definidas.

- As metas são negociadas, em vez de se basearem nos desejos dos stakeholders, nos limites fundamentais do processo e na melhoria das capacidades dos processos.

- Não há sistema organizado que quebre as metas de alto nível em subprocessos de níveis inferiores onde melhorias reais das atividades ocorrem, dificultando o alinhamento das metas intermediárias e das atividades dos empregados com as metas de alto nível.

- A não utilização do estado da arte em melhoria de processo.

- Falta de ligação entre o lado financeiro e o não financeiro no scorecard.

Goldszmidt (2003), a partir de uma revisão da literatura, identificou 14 fatores críticos para a implantação do balanced scorecard, os quais foram separados em dois grupos: fatores organizacionais e fatores relacionados ao processo de implantação e uso do BSC. Os fatores críticos do primeiro grupo relacionaram-se a processos organizacionais, cultura organizacional, estratégia e sistemas de informação. O segundo grupo apresentou fatores como: consciência da real finalidade do projeto e do esforço necessário; liderança e apoio da alta administração; equipe 
de projeto; individualização do projeto; abrangência do projeto e projeto-piloto; elaboração dos indicadores; simplicidade; comunicação e disseminação; dinâmica da aplicação do BSC e integração com os processos gerenciais.

Pode-se perceber que existem muitas barreiras à implantação de uma metodologia de gestão baseada no balanced scorecard, principalmente se não existir na empresa uma cultura de gestão estratégica no quadro gerencial (GALAS, 2004).

\section{Estudos relacionados de BSC e DEA}

Nesta seção, a partir da construção de um breve referencial teórico abrangendo as duas abordagens de avaliação mencionadas (BSC e DEA), apresenta-se a metodologia DEA-BSC.

Alguns estudos com a utilização dessas metodologias em separado para avaliação de desempenho podem ser encontrados na literatura.

Wang (2006) buscou mostrar o quanto as técnicas BSC e DEA eram eficientes em termos de informação gerada no processo de mensuração de performance. $\mathrm{O}$ autor desenvolveu ambos os métodos de forma isolada, demonstrando como o primeiro promovia incrementos no âmbito dos objetivos estratégicos e o segundo, eficiência técnica.

Além deste trabalho, muitos outros abordam a importância da utilização da DEA ou do BSC para avaliação de desempenho. A seguir, pode-se observar a junção desses métodos em alguns estudos, a fim de que as análises sejam potencializadas.

O primeiro a utilizar as metodologias de avaliação de desempenho DEA e BSC combinadas foi Rickards (2003). A proposta de utilização dessa metodologia decorreu da grande variedade de indicadores da ferramenta de avaliação de desempenho BSC, como uma alternativa para limitá-la. Segundo o autor, a diversidade de informações faz com seja complicado comparálas no processo de mensuração de performance. Com a DEA, as variáveis de análise escolhidas sob as perspectivas do BSC são tratadas em cada unidade, num universo reduzido, visto que a unidade eficiente passa a servir de referência para as demais e, assim, as melhorias são propostas sempre tendo como base a unidade $100 \%$ eficiente.

Eilat et al. (2006) propuseram a utilização do método DEA-BSC como uma alternativa superior a ambos os métodos de avaliação, se utilizados separados. A ideia foi embeber o BSC na metodologia DEA, de forma que a estrutura hierárquica estratégica de pesos do BSC fosse considerada por ela. O método, apresentado para avaliação de projetos de pesquisa e desenvolvimento (P\&D) em seus diferentes estágios (proposta, andamento e conclusão), foi construído tendo como base a integração da DEA a uma estrutura específica de BSC para projetos de $\mathrm{P} \& \mathrm{D}$ proposta por Stewart e adaptada pelos autores às condições do estudo. Essa 

processos internos, aprendizado e crescimento) e uma adicional, incerteza. Foram analisados cinquenta (50) projetos, através de onze variáveis output e duas input de três diferentes maneiras. A primeira utilizou DEA-CRS às variáveis dos indicadores de entrada e saída. A segunda, já com a metodologia proposta DEA-BSC, a partir do estabelecimento dos limites superior e inferior das perspectivas, conferiu importância relativa às variáveis. E na última, também com a metodologia DEA-BSC, os autores utilizaram o artifício de forçar os limites das perspectivas (30\%-70\% financeira; 2\%-12\% - incerteza; $6 \%-46 \%$ - as demais), conferindo maior importância à perspectiva financeira do que às demais, visto que ela possui maior peso nesse tipo de avaliação. Os resultados mostraram ser consistente, no processo de análise de desempenho, a metodologia integrada.

Chen e Chen (2007), em seu estudo, utilizaram a metodologia DEA-BSC tendo como objetivo a mensuração da eficiência da indústria de semicondutores de Taiwan. Para tanto, foram analisadas trinta (30) empresas. A escolha das variáveis foi feita com base nas perspectivas: financeira, clientes, processos internos, aprendizado e crescimento do BSC. Em cada uma delas foram selecionados indicadores que, posteriormente, seriam utilizados como inputs ou outputs, sob abordagem da DEA, para avaliação da performance daquele determinado aspecto. Assim, o BSC foi utilizado com o objetivo de ampliar a possibilidade de variáveis a serem utilizadas. Os resultados mostraram ser o aspecto financeiro o mais eficiente e, por isso, o de maior importância.

\section{Metodologia}

Esta pesquisa pode ser caracterizada, de acordo com Vergara (2004), como descritiva e quantitativa, pois procura-se, através da aplicação da análise envoltória de dados às informações de agências de um grande banco brasileiro, expor características a respeito da performance deste.

A amostra utilizada abrangeu 50 agências, o que torna o processo de amostragem não probabilístico. Como corresponde a um universo restrito, já que não foi utilizada a totalidade de agências desse banco, a pesquisa apresenta algumas limitações de inferência, o que, no entanto, não invalida seus resultados.

$\mathrm{O}$ estudo foi feito a partir de dados oriundos das perspectivas do BSC utilizadas pelo banco. De cada uma das agências, foram coletadas informações referentes às seguintes perspectivas: estratégia e operações, processos internos, comportamento organizacional, resultado econômico, satisfação dos clientes e impacto social, cada uma descrita abaixo. 
- Estratégia e operações (EO): representam o volume de operações financeiras e de crédito. A perspectiva engloba o volume de capital de crédito, linhas de investimento, operações de crédito em comércio exterior, transferências financeiras internacionais, captação, tarifas em serviços e número de salários pagos;

- Processos internos (PI): representam o índice de controle da unidade. A perspectiva engloba complice em processos administrativos e de crédito, processos contábeis e gestão de contas internas;

- Comportamento organizacional (CO): representa o aprendizado e qualidade do trabalho. A perspectiva engloba tempo, a qualidade do mesmo e a não utilização de horas extras (isso parte do que a empresa entende como qualidade de vida).

- Resultado econômico (RE): representa, em termos financeiros, o resultado obtido pela unidade. A perspectiva engloba os resultados advindos de serviços, tarifas, operações, seguridade, despesas; qualidade da carteira de crédito; previsão para devedores duvidosos; eficiência econômica;

- Clientes (CLI): essa perspectiva representa a satisfação dos clientes e engloba a satisfação dos clientes através de amostra aleatória não identificada.

- Sociedade (SOC): representa a participação das unidades bancárias na sociedade como incentivadoras de mudança ou mesmo de manutenção da realidade presente. A perspectiva engloba a participação da unidade em projetos sociais diversos, desde incentivo à horta orgânica, palestras sobre temas ambientais, manutenção de bibliotecas ou telecentros da fundação, entre outros.

As perspectivas EO, PI e CO foram utilizadas como inputs, por se acreditar que atuavam como recursos capazes de promover resultado econômico, satisfação dos clientes e impacto social. Assim, RE, CLI e SOC seriam os outputs. No entanto, todas as perspectivas, a princípio, tinham como característica serem do tipo "quanto maior, melhor" e, por isso, para que as primeiras fossem utilizadas como indicadores de entrada (do tipo "quanto menor, melhor"), foi necessário usar um artifício, que se resume na inversão das perspectivas (1/perspectiva).

Um grande número de diferentes variáveis, como as apresentadas acima, pode ser utilizado para avaliar a eficiência de unidades organizacionais, tais como agências bancárias, cada uma podendo ser vista como um vetor de desempenho que representa um diferente aspecto da performance. Esses seriam modelos de avaliação de performance monocriteriais.

O que se busca neste artigo é apresentar uma metodologia multidimensional, na qual seja possível avaliar o desempenho de bancos de modo multicriterial, ou seja, considerando de 
maneira integrada todos os vetores de desempenho apresentados, a partir das perspectivas do balanced scorecard.

Isso é feito através da utilização da análise envoltória de dados (DEA), que mostra o quão uma agência é eficiente no tratamento de seus inputs e outputs em relação às outras. Essa análise fornece um indicador que varia de 0 a 1 ou de $0 \%$ a $100 \%$, sendo que somente as agências bancárias que obtêm índice de eficiência igual a um é que são efetivamente eficientes, ou seja, fazem parte da fronteira eficiente. Em termos práticos, o modelo procura identificar a eficiência de uma agência comparando-a com os melhores desempenhos observados.

Lins e Meza (2000) ressaltam que um caminho intuitivo para introduzir DEA é por meio de forma de razão. Para cada DMU, procura-se obter uma medida de razão de todos os outputs sobre todos os inputs ou ainda de todos os inputs sobre todos os outputs. Ou seja, a modelagem procura encontrar os pesos ótimos $\mathrm{u}_{\mathrm{j}}$ e $\mathrm{v}_{\mathrm{i}}$ para a resolução de um dos seguintes problemas de programação matemática.

No modelo com orientação input, c é a unidade (DMU) que está sendo avaliada. O problema acima envolve a procura de valores para u e v, que são os pesos, de modo que maximize a soma ponderada dos outputs (output "virtual") dividida pela soma ponderada dos inputs (input "virtual") da DMU em estudo, sujeita à restrição de que esse quociente seja menor ou igual a um, para todas as DMUs. Essa função está sujeita à restrição de que, quando o mesmo conjunto de coeficientes de entrada e saída (os vários $\mathrm{v}_{\mathrm{i}} \mathrm{e} \mathrm{u}_{\mathrm{j}}$ ) for aplicado a todas as outras unidades de serviços que estão sendo comparadas, nenhuma unidade excederá 100\% de eficiência ou uma razão de 1,00 .

$$
\begin{aligned}
& \operatorname{Max} E_{c}=\frac{\sum_{j=1}^{s} u_{j} y_{j c}}{\sum_{i=1}^{m} v_{i} x_{i c}} \quad \text { Orientação input } \\
& \text { S.a.: } \quad \frac{\sum_{j=1}^{s} u_{j} y_{j k}}{\sum_{i=1}^{m} v_{i} x_{i k}} \leq 1, k=1,2, \ldots, c, \ldots, n \\
& \operatorname{MinEc}=\frac{\sum v_{i} x_{i c}}{\sum u_{j} y_{j c}} \quad \text { Orientação output } \\
& \text { S.a.: } \frac{\sum v_{i} x_{i k}}{\sum u_{j} y_{j k}} \geq 1, k=1,2, \ldots, c, \mathrm{~K}, n \\
& u_{j}, v_{i} \geq 0, \forall x, y \\
& u_{j} \geq 0, \forall j \text {, } \\
& v_{i} \geq 0, \forall i
\end{aligned}
$$

Já no modelo com orientação output, a eficiência é calculada pelo inverso da função objetivo, ou seja, eficiência $=1 /$ E. Esse problema define a relação dos inputs sobre os outputs, onde c é o índice da unidade que está sendo avaliada. Temos nesse problema as mesmas variáveis de decisão $\mathrm{u}_{\mathrm{x}} \mathrm{e} \mathrm{v}_{\mathrm{y}}$, porém queremos minimizar a soma ponderada dos inputs (input 
virtual) dividida pela soma ponderada dos outputs (output virtual) da DMU em estudo, sujeita à restrição de que esse quociente seja maior ou igual a 1, para todas as DMUs.

Segundo Coelli et al. (1998), esse é um problema fracionário (não linear) de programação matemática de difícil solução, mas que pode ser facilmente resolvido transformando-se a relação em uma função linear, simplesmente considerando-se o denominador da função objetivo igual a um. De acordo com Charnes et al. (1994), os modelos DEA-CRS-I e DEA-CRS-O podem, então, ser apresentados da seguinte maneira:

$$
\begin{array}{lll}
\text { Max } E_{c}= & \sum_{j=1}^{s} u_{j} y_{j} & \multicolumn{2}{c}{\text { Min } \sum_{i=1}^{m} v_{i} x_{i c}} \\
\text { Orientação input } & \text { Orientação output } \\
\text { S.a.: } & \sum_{i=1}^{m} v_{i} x_{i c}=1 & \text { S.a. }: \sum_{j=1}^{s} u_{j} y_{j c}=1 \\
& \sum_{j=1}^{s} u_{j} y_{j k}-\sum_{i=1}^{m} v_{i} x_{i k} \leq 0, k=1,2, \ldots, c, \ldots, n & \sum_{i=1}^{m} v_{i} x_{i k}-\sum_{j=1}^{n} u_{j} y_{j k} \geq 0, k=1, \mathrm{~K}, c, \mathrm{~K}, n \\
& u_{j,} v_{i} \geq 0, \forall x, y . & \\
& & u_{j}, v_{i} \geq 0, \forall x, y
\end{array}
$$

Os modelos DEA-CRS-I e DEA-CRS-O foram utilizados para a análise dos dados, pois têm-se três inputs e três outputs na análise. Logo, o modelo, além de avaliar o desempenho das agências bancárias, procura, em uma análise de benchmarking, melhorias nos níveis dos indicadores para se ter, ao mesmo tempo, duas óticas: melhorias dos inputs e melhorias dos outputs, em termos das transformações utilizadas.

Em relação à modelagem utilizada, obteve-se a eficiência de cada DMU através do uso de um software de DEA, apresentado por Meza et al. (2003), denominado SIAD (sistema integrado de apoio à decisão).

\section{Apresentação e análise dos resultados}

Às informações das agências bancárias sob análise (anexo 1) aplicou-se o modelo de avaliação de eficiência DEA-CRS, construído a partir da combinação das orientações output e input, tanto para se obter a eficiência de cada DMU, quanto para se analisar as mudanças nos níveis de outputs e/ou inputs nas agências ineficientes, para que se tornassem eficientes. O Quadro 1 mostra os indicadores de eficiência obtidos pela metodologia aplicada.

Pode-se verificar, quanto ao desempenho, que as agências 5, 6, 8, 9, 10, 11, 13, 16, 18, 20, 22, 25, 26, 29, 31, 32, 33, 34, 35, 36, 37, 38, 39, 42, 43 e 46 foram eficientes. Na verdade, essas unidades foram as que melhor combinaram os indicadores de EO, PI e CO, para a produção de RE, CLI e SOC. Nota-se, porém, que as demais agências, com exceção da 3, 4, 14 e 40, também apresentaram altos índices de eficiência (maiores que 90\%), embora não tenham 

um comportamento padrão das agências em relação às metas do banco.

\section{Quadro 1}

Eficiência das agências sob análise

\begin{tabular}{|c|c|c|c|c|c|}
\hline Agências & Eficiência & Agências & Eficiência & Agências & Eficiência \\
\hline A 1 & $94,71 \%$ & A18 & EFICIENTE & A35 & EFICIENTE \\
\hline A2 & $95,98 \%$ & A19 & $83,50 \%$ & A36 & EFICIENTE \\
\hline A3 & $83,50 \%$ & A20 & EFICIENTE & A37 & EFICIENTE \\
\hline A4 & $87,89 \%$ & A21 & $97,09 \%$ & A38 & EFICIENTE \\
\hline A5 & EFICIENTE & A22 & EFICIENTE & A39 & EFICIENTE \\
\hline A6 & EFICIENTE & A23 & $97,36 \%$ & A40 & $86,78 \%$ \\
\hline A7 & $97,79 \%$ & A24 & $95,98 \%$ & A 41 & $97,80 \%$ \\
\hline A8 & EFICIENTE & A25 & EFICIENTE & A42 & EFICIENTE \\
\hline A9 & EFICIENTE & A26 & EFICIENTE & A43 & EFICIENTE \\
\hline A10 & EFICIENTE & A27 & $93,30 \%$ & A44 & $95,24 \%$ \\
\hline A11 & EFICIENTE & A28 & $97,66 \%$ & A45 & $95,24 \%$ \\
\hline A12 & $93,30 \%$ & A29 & EFICIENTE & A46 & EFICIENTE \\
\hline A13 & EFICIENTE & A30 & $98,68 \%$ & A47 & $95,24 \%$ \\
\hline A14 & $71,37 \%$ & A31 & EFICIENTE & A48 & $98,73 \%$ \\
\hline A15 & $93,46 \%$ & A32 & EFICIENTE & A49 & $95,24 \%$ \\
\hline A16 & EFICIENTE & A33 & EFICIENTE & A50 & $95,24 \%$ \\
\hline A17 & $96,53 \%$ & A34 & EFICIENTE & - & - \\
\hline
\end{tabular}

Entre as unidades eficientes, pode-se observar (anexo 2) que apenas as agências 6, 8, 9, 31, 32, 33, 34, 35, 38 e 42 não necessitam de alterações em nenhum de seus indicadores. As outras unidades tidas como eficientes pelo modelo na verdade necessitam de algumas modificações. Assim, a análise de melhorias ajuda a entender e discriminar as unidades realmente eficientes e a mostrar as unidades que servem de benchmark para as outras (inclusive para aquelas tidas, inicialmente, como eficientes).

Com base na média de melhorias por variável, prossegue-se uma análise adicional orientada pelas perspectivas do BSC do banco. Nas perspectivas resultado econômico (RE) e sociedade (SOC) verificaram-se os piores resultados. Ambas relacionam-se a variáveis externas às agências e esse pode ser um dos fatores responsáveis por esse desempenho. Isso pode ser justificado pela diversidade dos ambientes em que as agências estão inseridas, apesar de atuarem num mesmo segmento de mercado. Com relação ao resultado econômico, por exemplo, diferentes e não previstas necessidades de esforços para captação e retenção de clientes podem estar sendo impostas a algumas agências, o que faz com que alternativas do tipo tarifas especiais, dentre outras, estejam sendo empregadas. No que diz respeito à sociedade, aspectos de desenvolvimento, em especial econômicos e culturais, podem fazer com que algumas agências, 
em função de características locais (localização geográfica), como proximidade de comunidades carentes, tenham a possibilidade de fazer mais projetos sociais do que outras que talvez tenham pouca ou nenhuma possibilidade.

Enquanto essas perspectivas se apresentaram como deficitárias, clientes e comportamento organizacional tiveram um bom desempenho médio, necessitando dos menores índices de melhoria. Percebeu-se na perspectiva clientes o reflexo do resultado econômico, que provavelmente tem mantido os níveis de satisfação dos clientes. Já com relação ao comportamento organizacional, pode-se dizer que seus índices são bons em função de ser uma perspectiva interna, mais ou menos homogênea entre as agências, definida pelo banco como uma meta a ser cumprida. Isso faz com que não existam grandes variações provocadas por agentes locais/externos.

Por último, ainda com base nos resultados, procedeu-se a uma análise adicional em relação às variáveis. Foi feita uma verificação dos pesos atribuídos aos vetores de desempenho, para se saber, com base em pesos iguais a zero, as variáveis que estavam sendo "desprezadas" na análise de desempenho.

A perspectiva resultado econômico foi a que apresentou o maior número de zeros nos pesos da modelagem DEA. Isso significa que esse é o indicador mais "desprezado" na análise de desempenho. Em linhas gerais, quando uma variável tem peso (u ou v) igual a zero, é naturalmente problemática na obtenção dos índices de eficiência. Como a modelagem procura os melhores índices de desempenho, dadas as características de inputs e outputs para cada DMU, esta atribui zero a toda variável que possa atrapalhar o objetivo de maximizar a eficiência. Isso pode ser explicado por ser essa a perspectiva de maior dispersão na amostra.

\section{Conclusões e considerações finais}

A eficiência é um dos aspectos que vêm governando, em maior ou menor grau, dependendo da organização, a atuação de unidades de negócios, principalmente em instituições financeiras no Brasil e no mundo, nos últimos anos, conforme mostram os estudos aqui referidos.

Numa análise dos resultados apresentados, as melhores agências são aquelas que possuem um desempenho multicriterial superior. Isso quer dizer que estas, possuindo desempenho relativo superior, podem ser utilizadas como benchmark para as demais. Por outro lado, agências que têm, em geral, baixos índices de desempenho, precisam de mudanças drásticas em seus indicadores.

Os resultados mostram que, apesar de a modelagem básica ter apontado 26 unidades eficientes, pôde-se observar, pela análise de benchmarking, que apenas dez agências são tidas 
como padrão de referência para as outras, pois estas possuem nível de desempenho de tal ordem que não necessitam de melhorias.

Além disso, uma análise das perspectivas proporcionou a visão de que o resultado econômico (RE) foi a variável problemática. Ou seja, existem padrões de referência e excelência de algumas agências de destaque que não estão sendo alcançados pelas outras agências. Isso faz com que essa seja uma variável, nas perspectivas do BSC, que o banco precisa homogeneizar em suas agências. Porém, visto sob outro aspecto, o RE se apresenta com um grande potencial de melhoria do sistema.

Os resultados deste estudo propõem uma nova percepção sobre a performance de unidades de negócios a partir da análise de indicadores que representem as perspectivas do BSC, que não se encontram disponíveis aos gestores em geral através dos tradicionais métodos de análise. Ou seja, a partir de informações que não estariam disponíveis pelas técnicas convencionais, os resultados da análise envoltória de dados (DEA) podem proporcionar melhores condições de competitividade aos bancos, pela melhoria da atuação de suas agências, principalmente quando interpretadas e usadas com os conhecimentos e julgamentos próprios da alta administração sobre suas operações.

O assunto não se encontra encerrado, pois ainda há muito a ser explorado dessa metodologia em gestão de instituições financeiras. Este mesmo trabalho deve ter continuidade através de uma visão melhor dos vetores de desempenho que mais contribuem para uma mensuração mais apurada da eficiência organizacional, aplicando-se a metodologia aqui apresentada e discutida combinada com outras metodologias, tais como a análise discriminante.

\section{Referências}

ATKINSON, A. A.; BANKER, R. D.; KAPLAN, R. S.; YOUNG, S. M. Contabilidade gerencial. São Paulo: Atlas, 2000.

BANKER, R. D.; CHARNES, A.; COOPER, W. W. Some models for estimating technical and scale inefficiencies in data envelopment analysis. Management Science, v. 30, n. 9, 1078-1092, 1984.

CHARNES, A.; COOPER, W. W.; RHODES, E. Measuring the efficiency of decision making units. European Journal Of Operational Research, v. 2, n. 6, 429-444, 1978.

CHARNES, A.; COOPER, W. W.; LEWIN, A. Y.; SEIFORD, L. M. Data envelopment analysis. 2. ed. Boston: KAP, 1994. 
CHEN, T.; CHEN, L. DEA performance evaluation based on BSC indicators incorporated: the case of semiconductor industry. International Journal of Productivity and Performance Management, v. 56, n. 4, p. 335-357, 2007.

COELLI, T.; RAO, D. S. P.; BALTESE, G. E. An introduction to efficiency and productivity analysis. Boston: KAP, 1998.

DAVIS, S.; ALBRIGHT, T. An investigation of the effect of balanced scorecard implementation of financial performance. Management Accounting Research, v. 15, n. 2, p. 135-153, 2004.

EILAT, H.; GOLANY, B.; SHTUB, A.. R\&Dproject evaluation: an integrated DEA and balanced scorecard approach. Omega, v. 36, n. 5, p. 895-912, 2006.

EPSTEIN, M.; MANZONI, J. F. Implementing corporate strategy: from tableaux de bord to balanced scorecards. European Management Journal, v. 16, n. 2, p. 190-203, 1998.

FARREL, M.J. The measurement of productive efficiency. Journal of the Royal Statistical Society, v. 120, series A, n. 3, p. 253-290, 1957.

GALAS, E. S. Fatores que interferem na implantação de um modelo de gestão estratégica baseado no balanced scorecard: estudo de caso em uma instituição pública. In: ENCONTRO NACIONAL DA ANPAD, 28, 2004, Curitiba. Anais do XXVIII EnANPAD. Curitiba: Anpad, 2004. CD Rom.

GOLDSZMIDT, R. G. B. Uma revisão de literatura dos fatores críticos para a implementação e uso do balanced scorecard. In: ENCONTRO NACIONAL DA ANPAD, 27, 2003, Atibais/SP. Anais do XXVII EnANPAD. Atibais/SP: Anpad, 2003. CD Rom.

KAPLAN, R.; NORTON, D. A estratégia em ação: balanced scorecard. Rio de Janeiro: Campus, 1997.

KIMURA, H.; SUEN, S. Ferramentas de análise gerencial baseadas em modelos de decisão multicriteriais. RAE eletrônica. v. 2, n. 1, 2003. Disponível em: <http://www.rac.com.br/ eletronica>. Acesso em: ago. 2007.

LINS, M. P. E.; MEZA, L. A. Análise envoltória de dados e perspectivas de integração no ambiente de apoio à decisão. Rio de Janeiro: Coppe/UFRJ, 2000.

MACEDO, M. A. S.; SILVA, F. F.; SANTOS, R. M. Análise do mercado de seguros no Brasil: uma visão do desempenho organizacional das seguradoras no ano de 2003. Revista Contabilidade \& Finanças, Edição Especial - Atuária, 2006.

MEZA, L. A.; BIONDI NETO, L; SOARES DE MELlO, J. C. C. B.; GOMES. E. G.; COELHO, P. H. G. SIAD - sistema integrado de apoio à decisão: uma implementação computacional de modelos de análise envoltória de dados. In: SIMPÓSIO DE PESQUISA OPERACIONAL DA MARINHA, 6, 2003, Rio de Janeiro. Anais do VI Spolm. Rio de Janeiro: Casnav, 2003. CD Rom.

NEELY, A. Measuring business performance. London: The Economist Books, 1998. 
NORREKLIT, H. The balance on the balanced scorecard - a critical analysis of some of its assumptions. Management Accounting Research, v. 11, p. 65-88, 2000.

PACE, E. S. U.; BASSO, L. F. C.; SILVA, M. A. Indicadores de desempenho como direcionadores de valor. Revista de Administração Contemporânea, v. 7, n. 1, p. 37-65, 2003.

RICKARDS, R. Setting benchmarks and evaluating balanced scorecards with data envelopment analysis. Benchmarking: an international journal, v. 10, n. 3, p. 226-245, 2003.

SCHNEIDERMAN, A. M. Why balanced scorecards fail. Journal of Strategic Performance Measurement, Edição Especial, p. 6, 1999.

SLACK, N.; CHAMBERS, S.; HARLAND, C.; HARRISON, A.; JOHNSTON, R. Administração da produção. 2 ed. São Paulo: Atlas, 2002.

VERGARA, S. C. Projetos e relatórios de pesquisa em administração. 5. ed. São Paulo: Atlas, 2004.

WANG, J. Corporate performance efficiency investigated by data envelopment analysis and balanced scorecard. Journal of American Academy of Business, p. 312, 2006.

ZHU, J. Multi-factor performance measure model with application to fortune 500 companies. European Journal of Operational Research, v. 123, n. 1, p. 105-124, 2000. 
Anexo 1 - Dados originais das agências

\begin{tabular}{|c|c|c|c|c|c|c|}
\hline Agências & RE & CLI & SOC & EO & $\mathbf{P I}$ & $\mathrm{CO}$ \\
\hline A1 & 295 & 420 & 50 & 333 & 564 & 500 \\
\hline A2 & 443 & 420 & 60 & 400 & 531 & 576 \\
\hline A3 & 476 & 420 & 30 & 468 & 466 & 500 \\
\hline A4 & 458 & 420 & 50 & 464 & 319 & 525 \\
\hline A5 & 535 & 420 & 40 & 405 & 459 & 600 \\
\hline A6 & 600 & 420 & 80 & 373 & 600 & 500 \\
\hline A7 & 506 & 420 & 70 & 402 & 418 & 585 \\
\hline A8 & 470 & 420 & 90 & 407 & 523 & 573 \\
\hline A9 & 478 & 420 & 130 & 415 & 584 & 413 \\
\hline A10 & 474 & 420 & 60 & 435 & 495 & 600 \\
\hline A11 & 354 & 420 & 50 & 397 & 516 & 600 \\
\hline A12 & 335 & 420 & 50 & 378 & 482 & 560 \\
\hline A13 & 335 & 420 & 50 & 378 & 520 & 600 \\
\hline A14 & 393 & 420 & 40 & 398 & 383 & 428 \\
\hline A15 & 524 & 420 & 60 & 494 & 541 & 549 \\
\hline A16 & 344 & 420 & 30 & 392 & 527 & 600 \\
\hline A17 & 402 & 420 & 50 & 384 & 559 & 579 \\
\hline A18 & 421 & 420 & 30 & 324 & 538 & 600 \\
\hline A19 & 394 & 420 & 40 & 413 & 443 & 501 \\
\hline A20 & 422 & 420 & 50 & 454 & 551 & 600 \\
\hline A21 & 455 & 420 & 50 & 442 & 533 & 582 \\
\hline A22 & 334 & 420 & 60 & 346 & 587 & 600 \\
\hline A23 & 479 & 420 & 40 & 545 & 558 & 557 \\
\hline A24 & 381 & 420 & 50 & 391 & 489 & 576 \\
\hline A25 & 570 & 420 & 50 & 474 & 417 & 600 \\
\hline A26 & 376 & 420 & 50 & 445 & 583 & 600 \\
\hline A27 & 492 & 420 & 40 & 399 & 550 & 558 \\
\hline A28 & 520 & 420 & 50 & 446 & 466 & 585 \\
\hline A29 & 480 & 420 & 40 & 448 & 527 & 600 \\
\hline A30 & 460 & 420 & 50 & 469 & 585 & 482 \\
\hline A31 & 570 & 420 & 40 & 384 & 522 & 600 \\
\hline A32 & 443 & 420 & 70 & 470 & 566 & 600 \\
\hline A33 & 508 & 420 & 70 & 403 & 419 & 600 \\
\hline A34 & 396 & 420 & 60 & 370 & 591 & 600 \\
\hline A35 & 509 & 420 & 60 & 573 & 571 & 600 \\
\hline A36 & 460 & 420 & 60 & 484 & 522 & 600 \\
\hline A37 & 486 & 420 & 50 & 454 & 411 & 600 \\
\hline A38 & 578 & 420 & 50 & 495 & 590 & 600 \\
\hline A39 & 338 & 420 & 60 & 458 & 578 & 600 \\
\hline $\mathrm{A} 40$ & 471 & 420 & 70 & 452 & 485 & 500 \\
\hline A41 & 377 & 420 & 40 & 428 & 581 & 543 \\
\hline A42 & 380 & 420 & 40 & 498 & 590 & 600 \\
\hline A43 & 374 & 420 & 40 & 417 & 575 & 600 \\
\hline A44 & 342 & 400 & 50 & 427 & 432 & 600 \\
\hline A45 & 530 & 400 & 40 & 496 & 560 & 600 \\
\hline A46 & 374 & 420 & 40 & 407 & 511 & 600 \\
\hline A47 & 439 & 400 & 50 & 415 & 574 & 600 \\
\hline A48 & 424 & 400 & 80 & 444 & 466 & 600 \\
\hline A49 & 360 & 400 & 50 & 423 & 580 & 600 \\
\hline A50 & 398 & 400 & 50 & 406 & 558 & 600 \\
\hline
\end{tabular}


Anexo 2 - Dados ideais (propostos) para as agências

\begin{tabular}{|c|c|c|c|c|c|c|}
\hline Agências & RE & CLI & SOC & EO & PI & CO \\
\hline A1 & 600 & 443 & 74 & 406 & 597 & 528 \\
\hline A2 & 462 & 438 & 63 & 417 & 572 & 599 \\
\hline A3 & 600 & 503 & 72 & 560 & 572 & 599 \\
\hline A4 & 559 & 478 & 68 & 527 & 574 & 599 \\
\hline A5 & 535 & 420 & 56 & 540 & 579 & 600 \\
\hline A6 & 600 & 420 & 80 & 373 & 600 & 500 \\
\hline A7 & 517 & 430 & 72 & 411 & 429 & 598 \\
\hline A8 & 470 & 420 & 90 & 407 & 523 & 573 \\
\hline A9 & 478 & 420 & 130 & 415 & 584 & 413 \\
\hline A10 & 474 & 420 & 60 & 482 & 495 & 600 \\
\hline A11 & 509 & 420 & 50 & 571 & 571 & 600 \\
\hline A12 & 530 & 450 & 54 & 404 & 584 & 599 \\
\hline A13 & 490 & 420 & 50 & 407 & 520 & 600 \\
\hline A14 & 600 & 589 & 82 & 558 & 552 & 599 \\
\hline A15 & 561 & 449 & 64 & 530 & 578 & 588 \\
\hline A16 & 539 & 420 & 46 & 392 & 527 & 600 \\
\hline A17 & 591 & 435 & 52 & 527 & 579 & 599 \\
\hline A18 & 421 & 420 & 49 & 454 & 538 & 600 \\
\hline A19 & 600 & 503 & 60 & 495 & 582 & 599 \\
\hline A20 & 440 & 420 & 60 & 454 & 578 & 600 \\
\hline A21 & 469 & 433 & 51 & 456 & 548 & 599 \\
\hline A22 & 425 & 420 & 60 & 393 & 592 & 600 \\
\hline A23 & 507 & 431 & 59 & 561 & 574 & 599 \\
\hline A24 & 495 & 438 & 52 & 407 & 519 & 599 \\
\hline A25 & 570 & 420 & 50 & 479 & 563 & 600 \\
\hline A26 & 560 & 420 & 50 & 484 & 583 & 600 \\
\hline A27 & 527 & 450 & 54 & 449 & 589 & 599 \\
\hline A28 & 532 & 430 & 54 & 458 & 549 & 599 \\
\hline A29 & 480 & 420 & 49 & 448 & 546 & 600 \\
\hline A30 & 510 & 426 & 51 & 476 & 593 & 584 \\
\hline A31 & 570 & 420 & 40 & 384 & 522 & 600 \\
\hline A32 & 443 & 420 & 70 & 470 & 566 & 600 \\
\hline A33 & 508 & 420 & 70 & 403 & 419 & 600 \\
\hline A34 & 396 & 420 & 60 & 370 & 591 & 600 \\
\hline A35 & 509 & 420 & 60 & 573 & 571 & 600 \\
\hline A36 & 460 & 420 & 60 & 484 & 576 & 600 \\
\hline A37 & 570 & 420 & 52 & 474 & 458 & 600 \\
\hline A38 & 578 & 420 & 50 & 495 & 590 & 600 \\
\hline A39 & 511 & 420 & 60 & 482 & 578 & 600 \\
\hline A40 & 576 & 484 & 81 & 521 & 563 & 576 \\
\hline A41 & 600 & 429 & 63 & 439 & 594 & 556 \\
\hline A42 & 380 & 420 & 40 & 498 & 590 & 600 \\
\hline A43 & 573 & 420 & 51 & 487 & 575 & 600 \\
\hline A44 & 532 & 420 & 53 & 483 & 600 & 600 \\
\hline A45 & 557 & 420 & 54 & 520 & 600 & 600 \\
\hline A46 & 455 & 420 & 55 & 407 & 590 & 600 \\
\hline A47 & 461 & 420 & 54 & 436 & 600 & 600 \\
\hline A48 & 456 & 405 & 81 & 450 & 560 & 600 \\
\hline A49 & 494 & 420 & 57 & 531 & 600 & 600 \\
\hline & 512 & 420 & 53 & 498 & 600 & 600 \\
\hline
\end{tabular}

